\title{
Single Nigrostriatal Dopaminergic Neurons Form Widely Spread and Highly Dense Axonal Arborizations in the Neostriatum
}

\author{
Wakoto Matsuda, ${ }^{1}$ Takahiro Furuta, ${ }^{1}$ Kouichi C. Nakamura, ${ }^{1,2}$ Hiroyuki Hioki, ${ }^{1}$ Fumino Fujiyama, ${ }^{1}$ Ryohachi Arai, ${ }^{3 \dagger}$ \\ and Takeshi Kaneko ${ }^{1,2}$ \\ ${ }^{1}$ Department of Morphological Brain Science, Graduate School of Medicine, Kyoto University, Kyoto 606-8501, Japan, ${ }^{2}$ Core Research for Evolutionary \\ Science and Technology, Japan Science and Technology Agency, Kawaguchi 332-0012, Japan, and ${ }^{3}$ Department of Anatomy, Shiga University of Medical \\ Science, Otsu 520-2192, Japan
}

The axonal arbors of single nigrostriatal dopaminergic neurons were visualized with a viral vector expressing membrane-targeted green fluorescent protein in rat brain. All eight reconstructed tyrosine hydroxylase-positive dopaminergic neurons possessed widely spread and highly dense axonal arborizations in the neostriatum. All of them emitted very little axon collateral arborization outside of the striatum except for tiny arborization in the external pallidum. The striatal axonal bush of each reconstructed dopaminergic neuron covered $0.45-5.7 \%$ (mean $\pm \mathrm{SD}=2.7 \pm 1.5 \%$ ) of the total volume of the neostriatum. Furthermore, all the dopaminergic neurons innervated both striosome and matrix compartments of the neostriatum, although each neuron's arborization tended to favor one of these compartments. Our findings demonstrate that individual dopaminergic neurons of the substantia nigra can broadcast a dopamine signal and exert strong influence over a large number of striatal neurons. This divergent signaling should be a key to the function of the nigrostriatal system in dopamine-based learning and suggests that neurodegeneration of individual nigral neurons can affect multiple neurons in the striatum. Thus, these results would also contribute to understanding the clinicopathology of Parkinson's disease and related syndromes.

Key words: dopamine; nigrostriatal; Parkinson's disease; reinforcement; striatum; tyrosine hydroxylase

\section{Introduction}

Dopamine-containing neurons are distributed in the retrorubral field (RRF), substantia nigra pars compacta $(\mathrm{SNc})$, and ventral tegmental area (VTA), which correspond to cell groups A8, A9, and A10, respectively, in the mammalian mesencephalon (Dahlstroem and Fuxe, 1964). These dopamine-containing neurons have a strong influence on emotion, motivation and cognitive processes mainly by the projection from the VTA to limbic forebrain areas, and on motor control chiefly by the projection from the SNc and RRF to the neostriatum (for review, see Joel and Weiner, 2000; Björklund and Dunnet, 2007). It has also been suggested that dopamine signal serves as a reinforcement signal in those regions (for review, cf. Schultz, 1998). The neostriatum,

Received Aug. 23, 2008; revised Nov. 28, 2008; accepted Nov. 30, 2008.

This work was supported by Grants-in-Aid for Scientific Research 16200025, 17650100, 18500262, 18700341, and 18700342, and Grant-in-Aid for Scientific Research on Priority Areas 17022020, 18019017, 18020013, and 20700315 from The Ministry of Education, Culture, Sports, Science, and Technology. We acknowledge A. M. Graybiel, M. Kimura, and J.P. Bolam for thoughtful comments on this manuscript.

${ }^{\dagger}$ Deceased, Aug. 25, 2008.

The authors declare no competing financial interests.

Correspondence should be addressed to Prof. Takeshi Kaneko, Department of Morphological Brain Science, Graduate School of Medicine, Kyoto University, Kyoto 606-8501, Japan. E-mail: kaneko@mbs.med.kyoto-u.ac.jp.

W. Matsuda's present address: Department of Anatomy, Shiga University of Medical Science, 0tsu 520-2192, Japan.

DO1:10.1523/JNEUROSCI.4029-08.2009

Copyright $\odot 2009$ Society for Neuroscience $\quad$ 0270-6474/09/290444-10\$15.00/0 being a main input nucleus of the basal ganglia, receives massive cortical and thalamic inputs and sends inhibitory projections to the external segment of the globus pallidus (GPe), entopeduncular nucleus [internal segment of the GP (GPi)] and substantia nigra pars reticulata $(\mathrm{SNr})$, the latter two structures being output nuclei of the basal ganglia circuit. Furthermore, the neostriatum is known to have a mosaic organization and be composed of patch/striosome and matrix compartments which are distinguishable from each other by the expression of neurochemical markers and by input-output organization (Graybiel and Ragsdale, 1978; Bowen et al., 1981; Herkenham and Pert, 1981; Gerfen, 1984, 1985, 1989; Gerfen et al., 1985, 1987a,b; Donoghue and Herkenham, 1986; Jimenez-Castellanos and Graybiel, 1987; Langer and Graybiel, 1989; Kaneko et al., 1995). In particular, neurons in the striosome compartment are known to send their inhibitory axons selectively to the SNc (Gerfen, 1985), whereas those in the matrix compartment project directly or indirectly to the SNr and GPi (Alexander et al., 1990).

Recently, we have developed a new recombinant viral vector which labels the infected neurons in a Golgi-stain-like manner (Furuta et al., 2001). This vector is based upon a replicationdefective Sindbis virus and designed to express green fluorescent protein (GFP) with a membrane-targeting palmitoylation signal of GAP-43 (palGFP) under the control of a powerful subgenomic promoter of the virus. Sindbis virus is known to have high infec- 

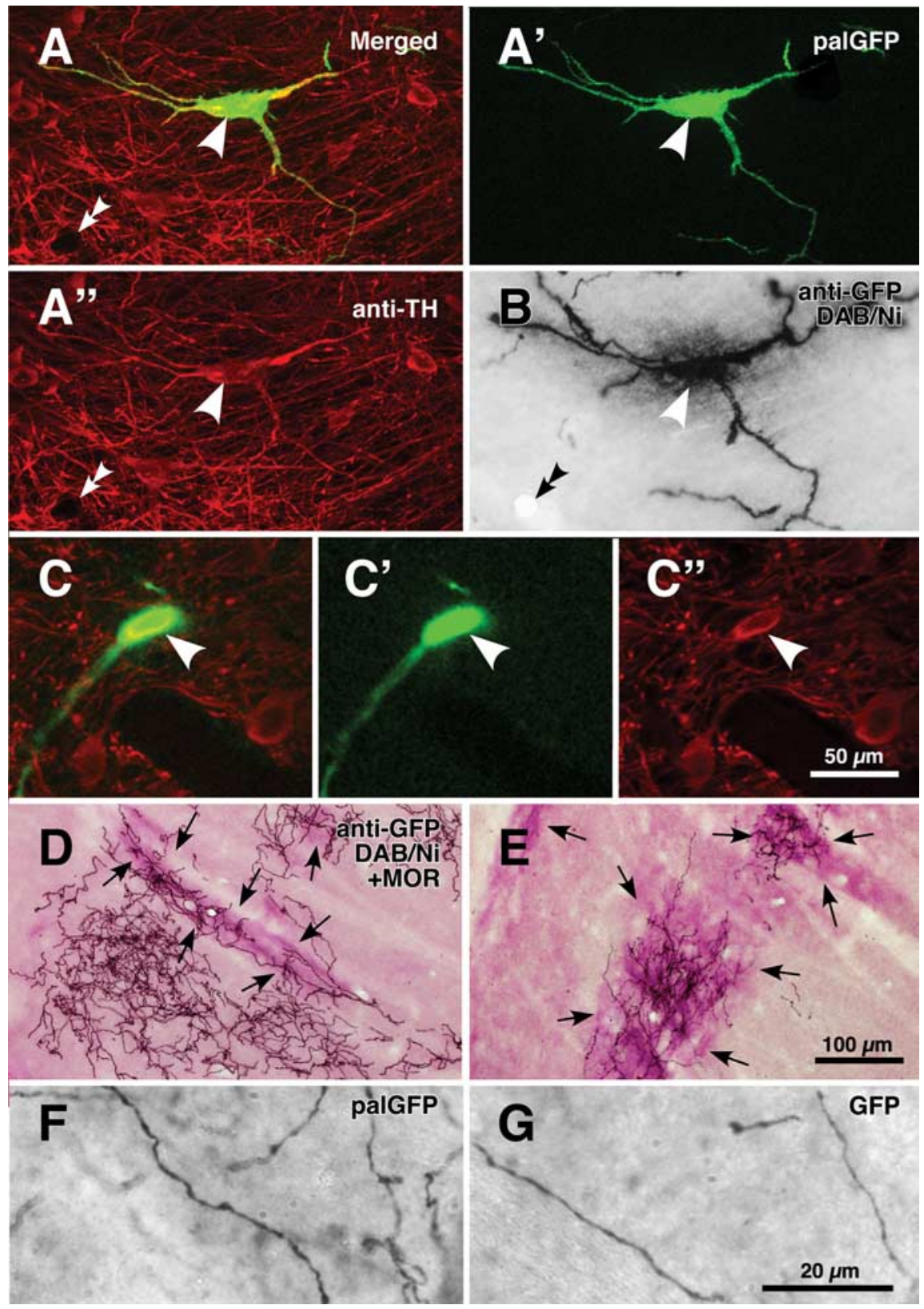

Figure 1. Single-neuron labeling with palGFP Sindbis virus vector. A nigral neuron showing green fluorescence of palGFP $(\boldsymbol{A}$, $\left.\boldsymbol{A}^{\prime}\right)$ was immunolabeled for TH $\left(\boldsymbol{A}, \boldsymbol{A}^{\prime \prime}\right)$, and further stained black by the immunoperoxidase method with anti-GFP antibody and diaminobenzidine/nickel reaction (DAB/Ni) $(\boldsymbol{B})$. Double arrowheads in $\boldsymbol{A}$ and $\boldsymbol{B}$ point to the same small vessel. Another example for palGFP-labeled TH-positive neurons is indicated by arrowheads in $\mathbf{C}-\boldsymbol{C}^{\prime \prime}$. The black-stained axon fibers of palGFP-labeled neurons densely innervated the striatum $(\boldsymbol{D}, \boldsymbol{E})$, being distributed both in the striosome/patch and matrix compartments $(\boldsymbol{D})$ or with preference for the striosome compartment $(\boldsymbol{E})$. MOR immunoreactivity was used to label the striosome compartments (violet-red areas indicated by arrows in $\boldsymbol{D}, \boldsymbol{E}$ ). palGFP-labeled axon fibers $(\boldsymbol{F})$ were compared with GFP-labeled fibers $(\boldsymbol{G})$ at a higher magnification. Scale bar in $\boldsymbol{C}^{\prime \prime}$ applies to $\boldsymbol{A}-\boldsymbol{C}^{\prime \prime}$, that in $\boldsymbol{E}$ applies to $\boldsymbol{D}, \boldsymbol{E}$, and that in $\boldsymbol{G}$ applies to $\boldsymbol{F}, \boldsymbol{G}$.

tion efficiency to a broad range of hosts, including neural cells (for review, cf. Xiong et al., 1989; Schlesinger, 1993; Piper et al., 1994; Strauss and Strauss, 1994; Lundstrom, 1999). Furthermore, since a large amount of palGFP is produced and distributed not only on the somatodendritic membrane but also on the axonal membrane of infected neurons (Furuta et al., 2001), the vector has been used as a highly sensitive anterograde tracer in the CNS (Nakamura et al., 2002, 2004, 2005; Ito et al., 2007). In the present study, we used the recombinant Sindbis virus vector as a tool for single-neuron labeling of dopaminergic neurons. We determined the dopaminergic characteristics of the infected neurons immu- nocytochemically, and visualized highly extensive axonal arborization of single nigrostriatal dopamine neurons. The axonal arborization was further examined in association with the striosome and matrix compartments of the neostriatum to clarify whether single dopamine signals exerted a selective influence upon the two different compartments in the neostriatum.

\section{Materials and Methods}

Animals. Forty-two adult male Wistar rats, weighing 250-310 g, were used. Experiments were conducted in accordance with the guidelines of the Committee for Animal Care and Use of the Graduate School of Medicine, Kyoto University, and those for Recombinant DNA Study, Kyoto University. All efforts were made to minimize the suffering and number of animals used in the present study.

Injection of Sindbis virus vector and fixation. Rats were anesthetized by ether inhalation and intraperitoneal injection of chloral hydrate (35 $\mathrm{mg} / 100 \mathrm{~g}$ body weight). Five to 10 infectious units of palGFP- or GFP-expressing Sindbis virus vector (Furuta et al., 2001) in $0.5 \mu$ l of PBS containing $2 \%$ bovine serum albumin were injected bilaterally into the $\mathrm{SNc}(5.3-5.5 \mathrm{~mm}$ posterior to the bregma, $2.2-2.4 \mathrm{~mm}$ lateral to the midline, and 7.2-7.7 $\mathrm{mm}$ deep from the brain surface) by pressure through a glass micropipette attached to Picospritzer III (General Valve Corporation). After survival for $36-42 \mathrm{~h}$, the rats were reanesthetized by intraperitoneal injection of chloral hydrate $(70 \mathrm{mg} / 100 \mathrm{~g}$ body weight), and perfused transcardially with 200 $\mathrm{ml}$ of PBS $[0.9 \%(\mathrm{w} / \mathrm{v})$ saline buffered with 5 mu sodium phosphate, $\mathrm{pH} 7.4$ ], followed by $200 \mathrm{ml}$ of $3 \%(\mathrm{w} / \mathrm{v}$ ) formaldehyde, $75 \%$ saturated picric acid and $0.1 \mathrm{M} \mathrm{Na}_{2} \mathrm{HPO}_{4}$ (adjusted with $\mathrm{NaOH}$ to $\mathrm{pH} 7.0$ ) over $30 \mathrm{~min}$. The brains were then removed, cut at the midline into two hemispheric blocks, and postfixed for $4 \mathrm{~h}$ at $4^{\circ} \mathrm{C}$ with the same fixative. After cryoprotection with 30\% (w/w) sucrose in PBS, the blocks were cut into $40-\mu \mathrm{m}$-thick parasagittal sections on a freezing microtome, and the sections were collected serially in PBS.

Immunofluorescence labeling for tyrosine hydroxylase. The sections including the injection site were observed under epifluorescent microscope Axiophot 2 (Zeiss) with an appropriate filter set (excitation, 450-490 nm; emission, 515-565 nm) for fluorescence of GFP to find SN neurons infected with the virus. All the following incubations described hereafter were performed at room temperature and followed by a rinse with PBS containing $0.3 \%(\mathrm{v} / \mathrm{v})$ Triton $\mathrm{X}-100$ and $0.02 \%(\mathrm{w} / \mathrm{v})$ merthiolate (PBS-X). The sections containing palGFP-positive SN neurons were incubated overnight with 1:200diluted mouse ascites containing anti-tyrosine hydroxylase $(\mathrm{TH})$ mono-

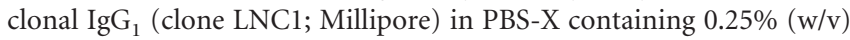
$\lambda$-carrageenan, $0.02 \%$ sodium azide and $1 \%(\mathrm{v} / \mathrm{v})$ donkey serum (PBS$\mathrm{XCD}$ ), and then for $4 \mathrm{~h}$ with $1 \mu \mathrm{g} / \mathrm{ml}$ AlexaFluor 594-conjugated anti[mouse IgG] goat antibody (Invitrogen) in PBS-XCD. Under the epifluorescence microscope with appropriate filter sets for GFP fluorescence and AlexaFluor 594 (excitation, 530-585 nm; emission, $\geq 615 \mathrm{~nm}$ ), 
Table 1. The axonal length of dopaminergic neurons in the striatum

\begin{tabular}{|c|c|c|c|c|c|}
\hline \multirow[b]{2}{*}{ TH-positive neuron } & \multirow[b]{2}{*}{ Position (tier) } & \multicolumn{3}{|c|}{ Length of axon fibers (in $\mu \mathrm{m})^{a}$} & \multirow{2}{*}{$\begin{array}{l}\text { Striosome } \\
\text { rate }(\%)^{b}\end{array}$} \\
\hline & & In matrix $(x)$ & In striosome $(y)$ & Total (z) & \\
\hline 1 & Dorsal & 638,332 & 141,601 & 779,933 & 18.2 \\
\hline 2 & Dorsal & 402,242 & 63,660 & 465,902 & 13.7 \\
\hline 3 & Dorsal & 130,981 & 7512 & 138,493 & 5.4 \\
\hline 4 & Dorsal & 245,004 & 33,982 & 278,986 & 12.2 \\
\hline 5 & Dorsal & 415,437 & 19,072 & 434,509 & 4.4 \\
\hline 6 & Ventral & 438,992 & 86,407 & 525,399 & 16.4 \\
\hline 7 & Ventral & 288,105 & 216,055 & 504,160 & 42.9 \\
\hline 8 & Ventral & 475,978 & 131,291 & 607,269 & 21.6 \\
\hline Average $\pm S D$ & & $379,384 \pm 155,772$ & $87,448 \pm 71,599$ & $466,831 \pm 195,198$ & $16.8 \pm 12.1$ \\
\hline
\end{tabular}

${ }^{a}$ The length of axon fibers was estimated by multiplying the length of axon fibers projected onto a parasagittal plane by $\pi / 2$ (when straight fibers having unit length and distributed in three-dimensionally random direction are projected to a plane, the mean length of projected fibers was $2 / \pi$ ).

${ }^{b} y / z \times 100$.

palGFP-labeled neurons were examined to determine whether they expressed immunoreactivity for TH (Fig. $1 A-A^{\prime \prime}$ ).

Double immunoperoxidase staining for GFP and $\mu$-opioid receptor. All the sections containing single palGFP-labeled and TH-immunopositive neurons were incubated overnight with a mixture of $0.4 \mu \mathrm{g} / \mathrm{ml}$ affinitypurified rabbit antibody to GFP (Tamamaki et al., 2000) and $2 \mu \mathrm{g} / \mathrm{ml}$ affinity-purified guinea pig antibody to $\mu$-opioid receptor (MOR) (Kaneko et al., 1995) in PBS-XCD. The incubation medium for the sections immunolabeled for TH further contained $10 \%$ (v/v) normal mouse serum to block the reaction with bound mouse antibody. After a rinse with PBS-X, the sections were incubated for $2 \mathrm{~h}$ with $10 \mu \mathrm{g} / \mathrm{ml}$ biotinylated anti-rabbit IgG donkey antibody (Millipore) in PBS-XCD, and then for $1 \mathrm{~h}$ with avidin-biotinylated peroxidase complex (ABC Elite; Vector) in PBS-X. After a rinse with PBS, the bound peroxidase was developed black by reaction for $10-30 \mathrm{~min}$ with $0.02 \%(\mathrm{w} / \mathrm{v})$ diaminobenzidine$4 \mathrm{HCl}, 10 \mathrm{~mm}$ nickel-ammonium sulfate and $0.0003 \%(\mathrm{v} / \mathrm{v}) \mathrm{H}_{2} \mathrm{O}_{2}$ in 50 $\mathrm{mm}$ Tris-HCl, pH 7.6 (Fig. $1 \mathrm{~B}$ ). The reaction was terminated by a rinse with PBS containing $2 \%(\mathrm{w} / \mathrm{v})$ sodium azide, followed by two rinses in PBS. Subsequently, the sections were further incubated for $2 \mathrm{~h}$ with 10 $\mu \mathrm{g} / \mathrm{ml}$ of biotinylated anti-guinea pig IgG donkey antibody (Millipore) in PBS-XCD, and then for $1 \mathrm{~h}$ with the avidin-biotinylated peroxidase complex in PBS-X. Finally, the second bound peroxidase was visualized violet-red by reaction for $10-30 \mathrm{~min}$ with $0.01 \%(\mathrm{w} / \mathrm{v})$ Trisaminophenol methane, $0.07 \%(\mathrm{v} / \mathrm{v}) p$-cresol and $0.003 \%(\mathrm{v} / \mathrm{v}) \mathrm{H}_{2} \mathrm{O}_{2}$ in 50 mu Tris-HCl, pH 7.6 (Fig. 1D,E) (Kaneko et al., 1994). All the above incubations and reactions were performed at room temperature. The stained sections were serially mounted onto the gelatinized glass slides, dried up, washed in running water, dried up again, cleared in xylene, and finally coverslipped with mounting medium MX (Matsunami).

Reconstruction of single dopamine neurons. The cell body, dendrites, and projecting main axon of stained dopamine neurons were reconstructed under a microscope attached with camera lucida apparatus. In particular, the striatal axon fibers were photographed with a digital camera attached to the microscope with a $20 \times$ objective, and then traced on the photograph with software CANVAS 9 (ACD Systems International) in a computer. The axon fibers in a section were reconstructed one by one onto a parasagittal plane, and superimposed to create the medial view in the computer. For the frontal and dorsal views, the extent of axon fibers in each $40-\mu \mathrm{m}$-thick section was superimposed on frontal and horizontal planes, respectively, with software ImageJ (NIH; http://rsb.info.nih.gov/ $\mathrm{ij} /$ ). The length of axons was obtained separately in the striosome and matrix compartments by calculating the length of traced lines with "Object Information" function in software Canvas 9.

Localization of cell bodies. The location of infected neurons was determined by cytoarchitecture. After tracing of infected neurons and axon fibers, the coverslips were removed from glass slides and the mounted sections were counterstained for Nissl with Neutral Red (Acros Organics). In addition, control parasagittal sections of rat mesencephalon were serially stained for Nissl and immunostained for calbindin D-28k and TH (supplemental Fig. 1, available at www.jneurosci.org as supplemental material). These control sections were used as a reference for defining

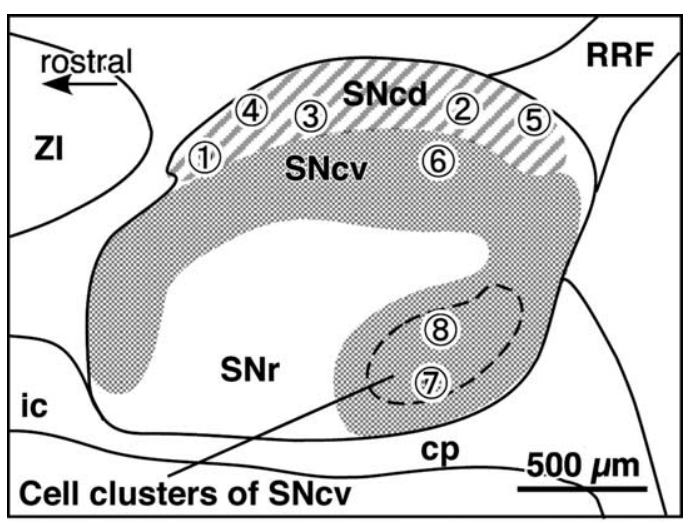

Figure 2. The distribution of the eight neurons labeled with palGFP Sindbis virus vector and immunopositive for tyrosine hydroxylase in the substantia nigra. The locations were projected onto a parasagittal plane $2.25 \mathrm{~mm}$ lateral to the midline. Each encircled numeral indicates the position of the neuron with the same number in Table 1. cp, Cerebral peduncle; ic, internal capsule; Zl, zona incerta.

ventral and dorsal tiers of the SNc by comparing cytoarchitecture with chemoarchitecture of calbindin and TH as described previously (Prensa and Parent, 2001).

Statistical analysis. The difference between dorsal and ventral tier of SNc dopamine neurons was determined using the two-tailed Student's $t$ test and two-tailed Mann-Whitney $U$ test.

\section{Results}

\section{Selection of neurons for single-cell tracing}

In the present study, we stereotaxically injected Sindbis virus vector for palGFP expression bilaterally into the $\mathrm{SN}$ of 42 rats, and thus of 84 hemibrains. The neurons for single-cell tracing were selected as follows: first, the hemiencephalic sections including the injection sites were examined under epifluorescence microscope. The hemibrains which contained only 1-10 palGFPlabeled SN neurons were selected. Seventy nigral neurons in 25 hemibrains were thereby processed for further analysis. Second, 70 palGFP-labeled neurons were examined for the expression of $\mathrm{TH}$ immunofluorescence, a marker for dopaminergic neurons in the SN (Fig. $1 A-A^{\prime \prime}, C-C^{\prime \prime}$ ). Twenty-one of 70 neurons were positive for TH immunoreactivity. Finally, the hemiencephalic sections containing these dopaminergic neurons were further stained by the immunoperoxidase method with anti-GFP antibody (Fig. $1 B, D-G$ ). Of the 21 stained neurons, the axons of 13 neurons were entangled or overlapped in the striatum with those of another neurons, and thus inseparable for reconstruction. 


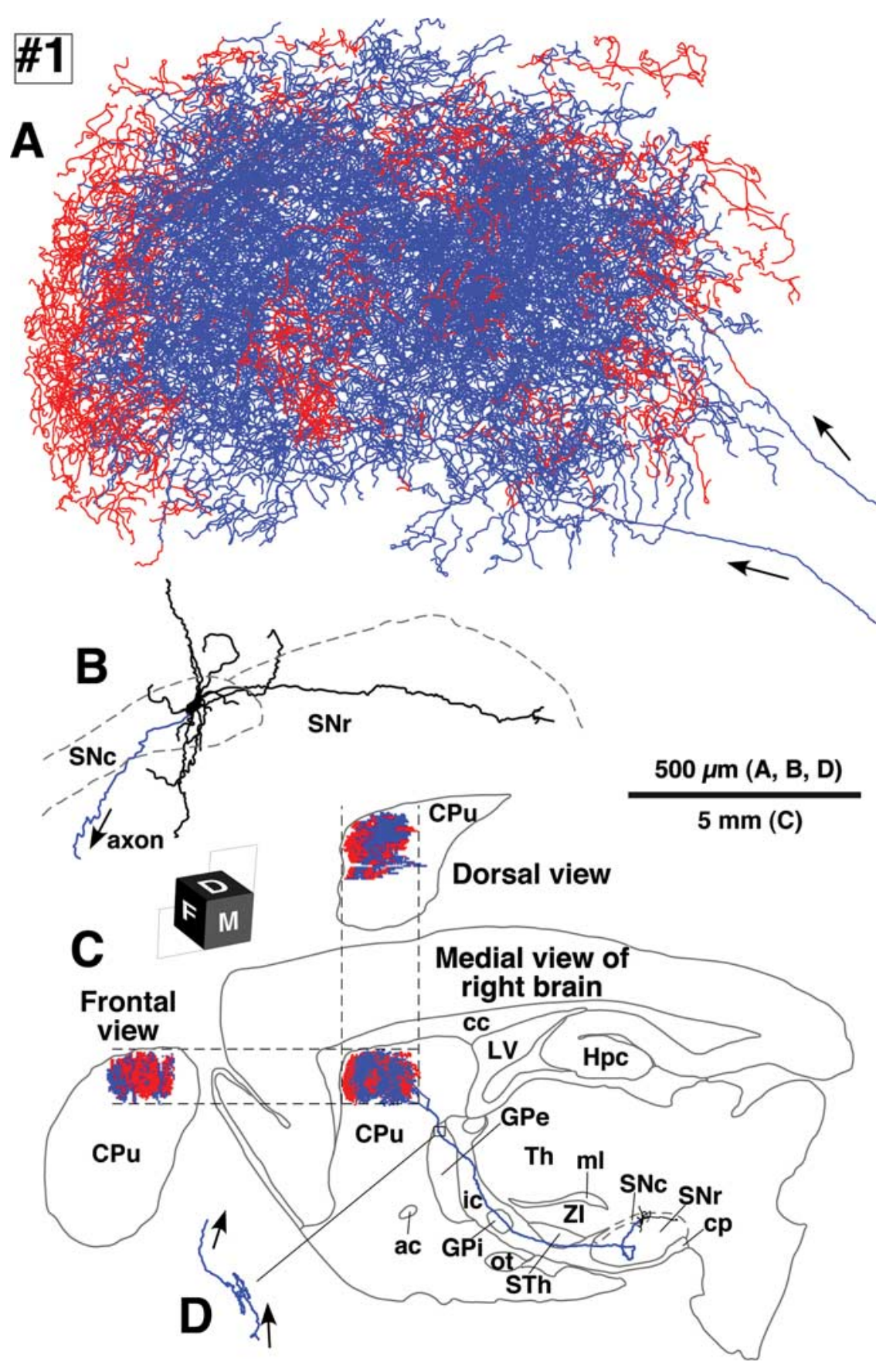

Figure 3. Camera lucida reconstruction of SNcd neuron \#1. $\boldsymbol{A}, \boldsymbol{B}$, The axon fibers in the striatum $(\boldsymbol{A})$ and dendrites $(\boldsymbol{B})$ in the $S \mathrm{NC}$ were projected onto a parasagittal plane and superimposed from the medial side. $\boldsymbol{C}$, The dorsal and frontal views of the intrastriatal axonal arborization were reconstructed and compared with the medial view. Red and blue lines in the striatum indicate the axon fibers located in the striosome and matrix compartments, respectively. Red fibers at the most rostral portion in $\boldsymbol{A}$ were mostly located in the MOR-positive subcallosal streak. $D$, The axon gave rise to only minor collaterals in the external segment of the GPe. ac, Anterior commissure; $c c$, corpus callosum; $\mathrm{Cp}$, cerebral peduncle; (Pu, caudate-putamen (neostriatum); Hpc, hippocampus; ic, internal capsule; LV, lateral ventricle; ml, medial lemniscus; ot, optic tract; STh, subthalamic nucleus; str, superior thalamic radiation; Th, thalamus; Zl, zona incerta.

Thus, only 8 neurons were selected for the present morphological analysis of single neurons. In 5 cases of them (Table 1, neurons \#2, 5-8), a hemibrain contained only one TH-immunopositive neuron. The other 3 neurons were found in a hemisphere, but fortunately separable from one another (Table 1, neurons \#1, 3, 4). Since TH-negative $\mathrm{SN}$ neurons (mainly located in the $\mathrm{SNr}$ ) had little, if any, axonal projection to the striatum, it was easy to separate axons of TH-positive neurons from those of TH-negative neurons.

These reconstructed neurons were subdivided into two groups based on the location of their cell bodies in the SNc (Fig. 2). By identifying the location of the cell bodies with Nissl counterstaining and by comparing Nissl cytoarchitecture with calbindin-immunoreactive structure in control parasagittal sections (supplemental Fig. 1, available at www.jneurosci.org as supplemental material), five neurons (Table 1, TH-positive neurons \#1-5) were classified as neurons of the dorsal tier of the $\mathrm{SNc}(\mathrm{SNcd})$, and three $(\# 6-8)$ were as neurons of the ventral tier $(\mathrm{SNcv})$. The region around each palGFP-positive neuron was darkly immunostained, probably due to the presence of extracellularly leaked palGFP (Fig. $1 B$ ), suggesting an extremely strong expression of protein by the subgenomic promoter of Sindbis virus. However, no spread to adjacent cell bodies was observed.

\section{Intrastriatal axons and striosome-matrix organization of the striatum}

Simultaneously with GFP immunoperoxidase staining, immunoreactivity for MOR was visualized in violet-red (Fig. $1 D, E$, arrows), and used as a marker for the striosome compartment of the striatum (Kaneko et al., 1995). As shown in Figure $1, D$ and $E$, some axon fibers of palGFPlabeled TH-positive SN neurons seemed to prefer the matrix or striosome compartment to the other compartment. These intrastriatal axons were relatively thick and seemed continuous to varicosities, making it difficult to count varicosities (Fig. $1 F$ ). Furthermore, axon fibers located in the striosome compartment showed little morphological difference compared with those in the matrix compartment.

To examine the effect of the palmitoylation signal on the morphology of intrastriatal axon fibers, we compared palGFPlabeled axon fibers and those labeled with unmodified green fluorescent protein (GFP). As shown in Figure 1, $F$ and $G$, there was no obvious difference between the two fibers. Since intrastriatal axon fibers were not completely labeled with GFP (supplemental Fig. 2, available at www. jneurosci.org as supplemental material), we could not compare global properties such as the total axonal length, but local characteristics between palGFP-labeled axon fibers and well labeled fibers with GFP. For example, the fiber width and interbranch interval of 80 randomly selected GFPlabeled axon fibers were $0.82 \pm 0.11 \mu \mathrm{m}$ and $31.2 \pm 19.4 \mu \mathrm{m}$, respectively. These data were not statistically different $(p \geq 0.72$ by the two-sided $t$ test) from those of 80 palGFP-labeled intrastri- 
atal axon fibers, the fiber width and interbranch interval of which were $0.83 \pm 0.13$ $\mu \mathrm{m}($ mean $\pm \mathrm{SD})$ and $30.9 \pm 21.6 \mu \mathrm{m}$, respectively (for further detail, see supplemental data, available at www.jneurosci. org as supplemental material). Thus, as far as we could compare, no significant difference was detected between the GFPlabeled and palGFP-labeled axon fibers.

\section{Axons of single nigrostriatal dopaminergic neurons}

The axons of all eight nigrostriatal dopaminergic neurons were isolated and reconstructed by camera lucida drawings as shown in Figures 3-7. The representative axonal distribution of neuron \#2 is shown in the photographs of supplemental Figure 3 , available at www.jneurosci.org as supplemental material. In all the reconstructed neurons, the main axon emerged from the cell body or proximal dendrite in the $\mathrm{SNc}$ without local axon collaterals around the cell body, then passed through the internal capsule, and then headed directly to the striatum. All but one of the axons emitted very short curly collateral projections in the GPe before entering the striatum (Figs. 3D, 5D-7D, neuron \#2 was the exception). No such collaterals were observed in the GPi. In contrast to the minimal collateral projections within extrastriatal structures, the individual nigrostriatal axons formed widely spread and highly dense axonal bushes within the striatum (Figs. $3 A-7 A, 3 C-7 C$ ). The axonal arborizations reconstructed in the striatum were more extended and much denser than those of the nigrostriatal axons described previously (Gauthier et al., 1999; Prensa and Parent, 2001), suggesting that the present method using the palGFPexpressing Sindbis virus vector was a highly sensitive and efficient technique for visualizing the arborization of single axons. The eight SN neurons had a total axonal length, on average, of $\sim 467,000 \mu \mathrm{m}$ (Table $1, \# 1-8$ ), whereas the seven representative $\mathrm{SN}$ neurons of the previous study (Prensa and Parent, 2001) had that of 36,000 $\mu \mathrm{m}$ (see supplemental Table 1, estimated from the published data, available at www.jneurosci.org as supplemental material), $<1 / 10$ of the present data. This indicates that the axonal arborization of the previous studies was largely underestimated. The intrastriatal axonal arborizations of neurons \#1, 2, 5, 7 and 8 innervated mainly the dorsal part of the striatum, and those of neurons \#3, 4 and 6 mainly the middle and ventral parts of the striatum, sometimes including the border between the dorsal striatum and the accumbens nucleus.

The five SNcd neurons had total axonal lengths of 140,000780,000 $\mu \mathrm{m}$ (Table 1, \#1-5). The typical wide spread arborizations of intrastriatal axons was observed in dopamine neurons \#1, $\# 2$, \#4 and \#5, and those of neurons \#1 and \#2 were reconstructed in Figures 3 and 4, respectively. The arbors occupied oval volumes that extended $1.5-2.0 \mathrm{~mm}$ rostrocaudally, $0.7-1.0 \mathrm{~mm}$ dor- soventrally and $1.0-1.5 \mathrm{~mm}$ mediolaterally. The total axonal lengths of these four neurons ranged from 280,000-780,000 $\mu \mathrm{m}$ (Table 1). The striatal volume covered with the dense axonal plexus was estimated to be $0.09-1.12 \mathrm{~mm}^{3}$ (Table 2 ) and reached a maximum of $5.7 \%$ of the total neostriatal volume on one side $\left(19.81 \pm 0.63 \mathrm{~mm}^{3}, n=3\right)$. SNcd dopamine neuron \#3 was an exceptional case with a smaller and mediolaterally flat axonal bush, shown in Figure 5. This arbor formed a small terminal field in the fundus of the caudoputamen as a main bush with a large diameter of $\sim 500 \mu \mathrm{m}$ and a smaller bush arbor extending rostroventral to the main bush. Although this neuron had the smallest axonal bushes in the present study, the total axonal length in the striatum was $140,000 \mu \mathrm{m}$ (Table 1).

The three SNcv neurons had total axonal lengths of 500,000$610,000 \mu \mathrm{m}$ (Table $1, \# 6-8$ ). Two of them (\#7 and \#8) are illustrated in Figures 6 and 7, respectively. The arbors of SNcv neurons \#6 and \#8 formed single oval volumes with a major diameter of $>1 \mathrm{~mm}$ as had shown in SNcd dopamine neurons. Neuron \#7 formed two axonal bushes, large and small ones, similar to SNcd 
Table 2. Volume occupied by TH-positive axons and axon fiber density in the striatum

\begin{tabular}{|c|c|c|c|c|c|c|}
\hline \multirow[b]{2}{*}{ TH-positive neuron } & \multicolumn{3}{|c|}{ Volume occupied by axons (in $\left.\mathrm{mm}^{3}\right)^{a}$} & \multicolumn{2}{|c|}{ Axon density (in $\left.\mu \mathrm{m} / \mathrm{mm}^{3}\right)^{b}$} & \multirow{2}{*}{$\begin{array}{l}\text { Striosome- } \\
\text { matrix den- } \\
\text { sity ratio } \\
([y / b] /[x / a])\end{array}$} \\
\hline & In matrix (a) & $\begin{array}{l}\text { In striosome } \\
\text { (b) }\end{array}$ & Total (c) & $\begin{array}{l}\text { In matrix } \\
(x / a)\end{array}$ & $\begin{array}{l}\text { In striosome } \\
(y / b)\end{array}$ & \\
\hline 1 & 0.94 & 0.18 & 1.12 & 678 & 802 & 1.18 \\
\hline 2 & 0.59 & 0.07 & 0.66 & 681 & 854 & 1.25 \\
\hline 3 & 0.08 & 0.01 & 0.09 & 1691 & 1130 & 0.67 \\
\hline 4 & 0.25 & 0.03 & 0.28 & 997 & 1155 & 1.16 \\
\hline 5 & 0.59 & 0.06 & 0.65 & 703 & 294 & 0.42 \\
\hline 6 & 0.53 & 0.06 & 0.59 & 843 & 1370 & 1.64 \\
\hline 7 & 0.42 & 0.07 & 0.49 & 693 & 3139 & 4.53 \\
\hline 8 & 0.36 & 0.08 & 0.44 & 1310 & 1715 & 1.31 \\
\hline Average \pm SD & $0.47 \pm 0.26$ & $0.07 \pm 0.05$ & $0.54 \pm 0.30$ & $950 \pm 371$ & $1307 \pm 851$ & $1.52 \pm 1.27$ \\
\hline
\end{tabular}

In a section, the matrix or striosome area occupied by the axon fibers was measured in a polygon circumscribed to the axonal arborization. The volume in a section was calculated by multiplying those areas with the thickness of the section and these volumes in single sections were summed up to the whole volume.

${ }^{b}$ Data $x$ and $y$ were taken from Table 1.

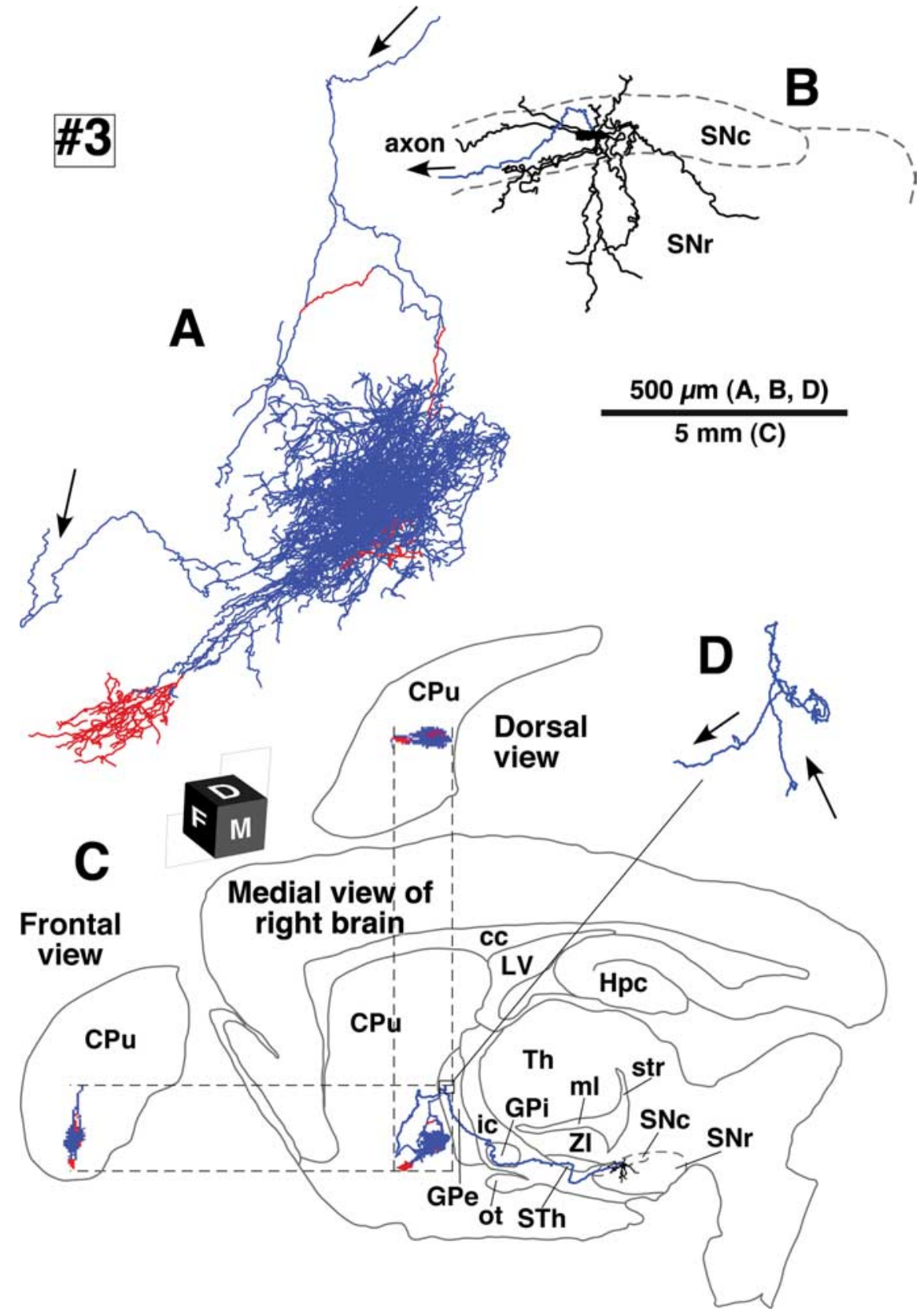

Figure 5. Camera lucida reconstruction of SNcd neuron \#3. Of the eight neurons examined in the present study, this neuron had the smallest axonal arborization in the striatum. See the legend of Figure 3 for further detail. neuron \#3, although the arborization was much bigger than that of neuron \#3.

Comparison between single-axon arbors innervating the striosome and matrix compartments of

the neostriatum

Most intrastriatal axon fibers of the palGFP-labeled dopaminergic neurons were distributed nonselectively in the striosome and matrix compartments (Fig. $1 D)$, but some fibers appeared to innervate either the striosome or the matrix compartment preferentially (Fig. $1 E$ ). Because the labeled fibers possessed many ambiguous varicosities in the striatum (Fig. $1 F, G$ ) and synaptic specializations are not often found at large varicosities but frequently at the thinner parts of dopaminergic axons (Pickel et al., 1981; Arluison et al., 1984; Freund et al., 1984; Zahm, 1992), the intrastriatal length of axon fibers, instead of the density (or the number) of varicosities, was compared between striosome and matrix compartments to evaluate quantitatively the contribution of single dopaminergic neurons to each compartment.

The striosome compartment including the subcallosal streak has been reported to occupy $10-13 \%$ of the total neostriatal volume in the rat (Johnston et al., 1990; Desban et al., 1993). Two SNcd neurons \#3 and $\# 5$ sent only $4-5 \%$ of their axon fibers to the striosome compartment (Table 1), and thus appeared to prefer the matrix compartment over the striosome compartment. In contrast, all three $\mathrm{SNcv}$ neurons preferred the striosome compartment, although proportionally the striosome innervation constituted only a minority of their total axonal length (16$43 \%)$. On average, five SNcd neurons sent $10.8 \pm 5.2 \%$ (mean $\pm \mathrm{SD}$ ) of axon fibers to striosomes, and three $\mathrm{SNcv}$ neurons 
projected $27.0 \pm 11.5 \%$ of their axonal arbors to striosomes. The $p$-value of difference between the two groups was 0.0554 by a two-tailed Student's $t$ test, just failing statistical significance. We further examined whether or not SNcd and SNcv neurons showed the difference in local preference for either compartment by evaluating their axonal preference in the regions where the axon fibers were distributed (Table 2). We separately measured the striosome and matrix volumes which were covered by the projecting axon fibers, and then calculated the axon fiber density in the striosome and matrix compartments. In 3 of the $5 \mathrm{SNcd}$ neurons and all of the 3 $\mathrm{SNcv}$ neurons, the axon fiber density in the striosome compartment was higher than that in the matrix compartment (Table 2, striosome-matrix density ratio of $>1$ ). Furthermore, the striosome-matrix density ratio of $\mathrm{SNcv}$ neurons were significantly higher than that of SNcd neurons ( $p=0.0357$ by the two-tailed $U$ test). Thus the latter comparison partly supported the previously reported finding that $\mathrm{SNcv}$ neurons innervate the striosome/patch compartment and that SNcd neurons project preferentially to the matrix compartment (Gerfen et al., 1987a).

Notably, even for a given nigrostriatal neuron, the axonal preference for compartments was different between its axonal bushes. SNcd neuron \#3 and SNcv neuron \#7 both had an isolated small axonal bush that were preferentially distributed in striosomes, despite an overall wide distribution of the entire axon (Figs. 5, 6). This pattern indicates that the target preference of a single axon could be different depending on the striatal terminal fields it innervates, and that dopamine-containing innervation might not be so simple as hypothesized in the simple dopamine-dependent learning model of the basal ganglia circuit.

In summary, although each nigrostriatal dopamine neuron showed some preference for the striosome or matrix compartment, all the dopamine neurons innervated both the striosome and matrix compartments. Furthermore, even a single dopamine axon altered its preference for the compartments in a field-byfield manner within the striatum.

\section{Discussion}

Analysis of the entire axonal arborizations of single dopaminergic nigrostriatal neurons with a new virus tracer demonstrated that the intrastriatal arbors of nigrostriatal neurons were much wider and denser than previously reported (Gauthier et al., 1999; Prensa and Parent, 2001), where single nigrostriatal axons were examined by a labeling method with a small injection of biotinylated dextran-amine into the rat SN. Moreover, although some axon arbors exhibited local preferences for one or the other compartment, all the single nigrostriatal axons innervated both striosome and matrix compartments of the striatum. A further critical finding in the present study is that, except for negligible arbors in the GPe, dopaminergic neurons here had very little arborization outside of the striatum.

Compared with previous reports on the nigrostriatal projection (Gauthier et al., 1999; Prensa and Parent, 2001), the largest difference found was the extremely high density of the intrastriatal arborization of the nigrostriatal axons we reconstructed. This difference may be a consequence of much higher sensitivity of the present single-cell tracing method using the palGFP Sindbis virus vector. It is possible that palGFP expression might have affected the axonal arborization of dopamine neurons, given that overexpression of GFP with a palmitoylation signal of GAP-43 N terminus [1-14] was reported to induce filopodia formation on the surface membrane of COS-7 cells and on the dendrites of cultured hippocampal neurons (Gauthier-Campbell et al., 2004). However, this possibility is unlikely for the following reasons (see also supplemental data, available at www.jneurosci.org as supplemental material). (1) The effect reported was on dendritic membranes, but not on axonal membranes. In the present study, neither filopodia-like nor growth cone-like structures were observed 


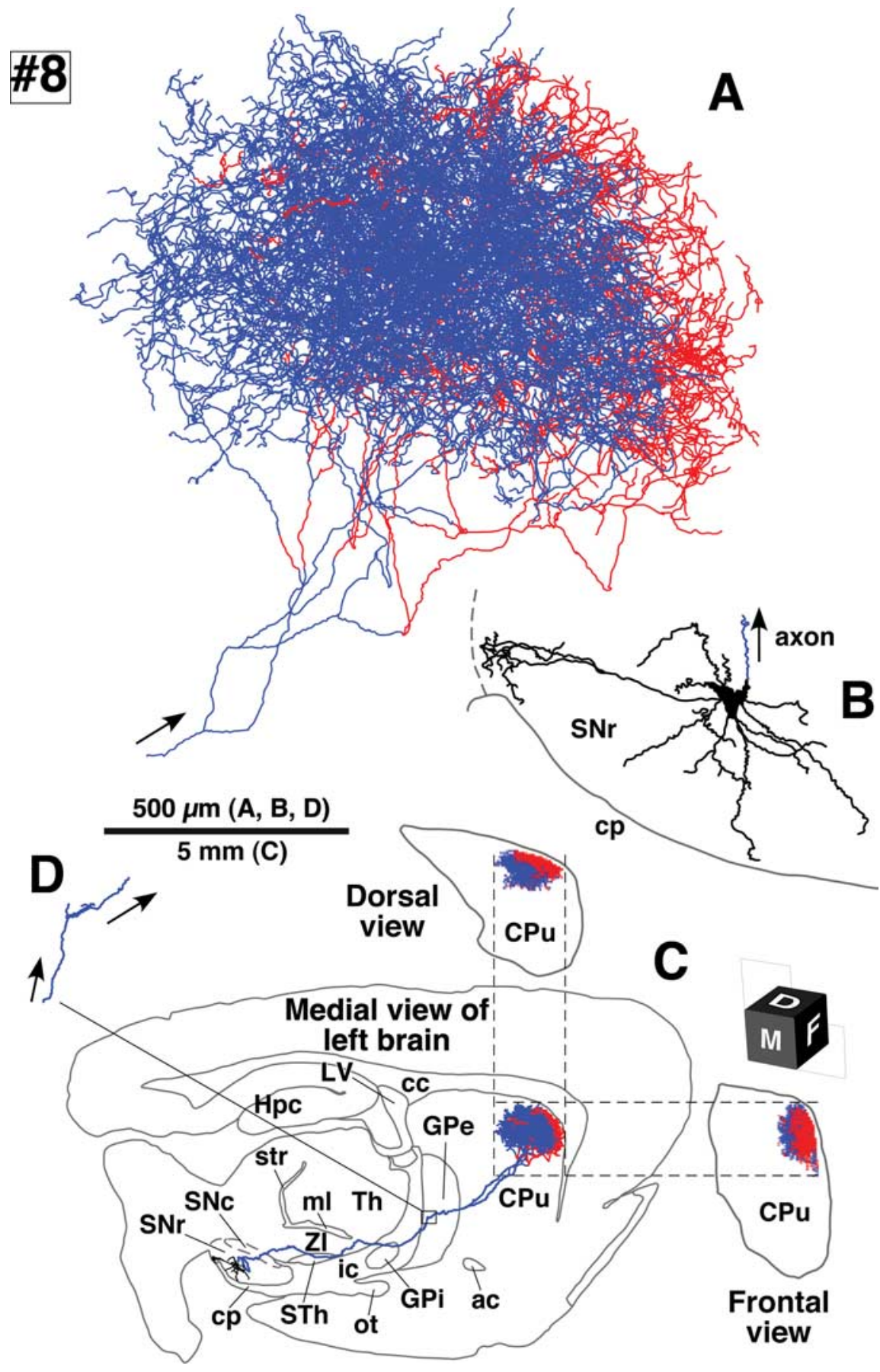

Figure 7. Camera lucida reconstruction of SNcv neuron \#8. Although the cell body of this neuron was located at the cell cluster region of the $S N c v$, the axonal arborization was very similar to that of SNcd neuron \#1 (Fig. 3). See the legend of Figure 3 for detail.

that palGFP expression seemed to have little, if any, morphological effect on the dopaminergic axons, and thus that naive nigrostriatal dopaminergic neurons may have as widely spread and highly dense axonal arborization in the neostriatum as the neurons infected with palGFP Sindbis virus.

A second major difference from previous findings concerns the nigrostriatal innervation of striosomes and matrix. The topography of the substantia nigra itself has been reported to provide the organization of the nigrostriatal projection, whereby neurons in the SNcd project chiefly to the matrix, whereas those in the $\mathrm{SNcv}$ send axons mainly to the striosome/ patch compartment in the rat brain (Gerfen et al., 1987a). In addition, differential nigrostriatal projections in association with striosome and matrix compartments have been reported in cat and primate brains (Jimenez-Castellanos and Graybiel, 1987; Langer and Graybiel, 1989). A segregation of these projections was partially supported by the present findings. There was some preference of SNcd neurons for the matrix compartment and some preference of SNcv neurons for striosomes, but we found that single SNcd or SNcv neurons basically innervated both the striosome and matrix compartments. This means that identical dopamine signals can be sent simultaneously to both striosome and matrix neurons.

Some reports favor a projection from striosomes to SNc dopaminergic neurons, although a recent report on monkey brain is in disagreement (Levesque and Parent, 2005): Favoring striosome-to-SNc projections are the findings that fluorescent retrograde tracers injected into rat mesencephalon including the SNc but not the SNr, mostly retrogradely labeled neurons in the striosome/patch compartment (Gerfen, 1985); and immunoreactivity for substance $\mathrm{P}$, which was produced by both patch and matrix neurons, is distributed mainly in the neuropil of the $\mathrm{SNr}$, whereas immunoreactivity for preprodynorphin, expressed more intensely in striosome

on axon fibers of TH-positive neurons. (2) Even on the dendritic membrane of palGFP-expressing neurons, we detected no morphological differences compared with the previous morphological studies of SNc neurons (Juraska et al., 1977; Preston et al., 1981; Tepper et al., 1987). (3) In a comparative study using GFP Sindbis virus vector, GFP-positive nigrostriatal axon fibers showed characteristics similar to those of palGFP-positive fibers, although the axon fibers were not completely visualized throughout their arborization (supplemental Fig. 2, available at www. jneurosci.org as supplemental material). No difference in the width or interbranch interval of axon fibers was detected between palGFP- and GFP-labeled axon fibers. Therefore, we conclude neurons, is distributed more densely in $\mathrm{SNc}$ neuropil than $\mathrm{SNr}$ neuropil (Lee et al., 1997). Thus, it seems most likely that striosomes contribute to the production of a dopamine signal by SNc neurons (Schultz, 1998; Doya, 2000). This dopamine signal is sent back to the neostriatum for the long-term modification of corticostriatal and/or thalamostriatal synapses (Calabresi et al., 2000, 2007), resulting in the updates of responsiveness of striatal neurons. The present results indicate that a large number of matrix and striosome neurons are affected by the single dopamine signal emitted by a single SNc neuron.

The present results indicate that, on average, $2.7 \%$ of neostriatal neurons (Table 2) $\left(0.54 \mathrm{~mm}^{3} / 19.81 \mathrm{~mm}^{3}=0.027\right)$ and, at the 
maximum, $5.7 \%$ of neurons $(1.12 / 19.81=0.057)$ are under a strong influence of a single dopamine neuron. Since the rat neostriatum contained 2,790,000 neurons in one side (Oorschot, 1996), and the volume innervated by a single dopamine neuron was, on average, $2.7 \%$ of the total neostriatal volume in the present study, $\sim 75,000$ striatal neurons are estimated to be influenced by a single dopaminergic neuron. The whole striatum might not need to receive a single dopamine signal, because the basal ganglia circuit is functionally segregated into several parallel circuits such as motor, oculomotor, prefrontal, and limbic circuits (for review, see Alexander et al., 1990). However, it is known that electrical synapses are formed between SNc dopaminergic neurons (Grace and Bunney 1983; Vandecasteele et al., 2005), suggesting a mechanism for synchronization or coactivation of dopaminergic neurons. If a single dopamine signal covers the whole striatum, the present results indicate that at least 37 dopaminergic neurons $(19.81 / 0.54 \approx 37)$ have to synchronize and convey the same dopamine signal.

Because the rat SNc contained 3500-7200 neurons in one side (Andén et al., 1966; Halliday and Tork, 1986; Oorschot, 1996), it is estimated that a neuron in the rat neostriatum might be under the influence of 95-194 dopaminergic neurons on average $(3500-7200 \div(19.81 / 0.54) \approx 95-194)$. This highly overlapping innervation of dopaminergic neurons raises the question of its necessity. The question might partially be answered by the fact that animals have many different kinds of reward-based signals, such as those related to appetitive and sexual behaviors. However, since it is unlikely that animals have a hundred kinds of reward-based signals, another justification seems necessary for this redundancy. The large margin of safety in dopaminergic neurons has been reported in human brains diagnosed as idiopathic Parkinson's disease, as extensive loss $(>70 \%)$ of neurons in the SNc occurs in most patients before parkinsonian symptoms appear (Hirsch et al., 1988). Since neurons are labile elements and are relatively easily lost from many causes, it is a good strategy for the system composed of such unreliable elements to maintain a high level of redundancy. Thus our findings have important implications for an understanding of the clinicopathology of Parkinson's disease and related neurodegenerative disorders.

\section{References}

Alexander GE, Crutcher MD, DeLong MR (1990) Basal gangliathalamocortical circuits: parallel substrates for motor, oculomotor, "prefrontal" and "limbic" functions. Prog Brain Res 85:119-146.

Andén NE, Hfuxe K, Hamberger B, Hökfelt T (1966) A quantitative study on the nigro-neostriatal dopamine neuron system in the rat. Acta Physiol Scand 67:306-312.

Arluison M, Dietl M, Thibault J (1984) Ultrastructural morphology of dopaminergic nerve terminals and synapses in the striatum of the rat using tyrosine hydroxylase immunocytochemistry: a topographical study. Brain Res Bull 13:269-285.

Björklund A, Dunnett SB (2007) Dopamine neuron systems in the brain: an update. Trends Neurosci 30:194-202.

Bowen WD, Gentleman S, Herkenham M, Pert CB (1981) Interconverting $\mathrm{mu}$ and delta forms of the opiate receptor in rat striatal patches. Proc Natl Acad Sci U S A 78:4818-4822.

Calabresi P, Centonze D, Gubellini P, Marfia GA, Pisani A, Sancesario G, Bernardi G (2000) Synaptic transmission in the striatum: from plasticity to neurodegeneration. Prog Neurobiol 61:231-265.

Calabresi P, Picconi B, Tozzi A, Di Filippo M (2007) Dopamine-mediated regulation of corticostriatal synaptic plasticity. Trends Neurosci 30:211-219.

Dahlstroem A, Fuxe K (1964) Evidence for the existence of monoaminecontaining neurons in the central nervous system. I. Demonstration of monoamines in the cell bodies of brain stem neurons. Acta Physiol Scand Suppl 232:1-55.
Desban M, Kemel ML, Glowinski J, Gauchy C (1993) Spatial organization of patch and matrix compartments in the rat striatum. Neuroscience 57:661-671.

Donoghue JP, Herkenham M (1986) Neostriatal projections from individual cortical fields conform to histochemically distinct striatal compartments in the rat. Brain Res 365:397-403.

Doya K (2000) Complementary roles of basal ganglia and cerebellum in learning and motor control. Curr Opin Neurobiol 10:732-739.

Freund TF, Powell JF, Smith AD (1984) Tyrosine hydroxylaseimmunoreactive boutons in synaptic contact with identified striatonigral neurons, with particular reference to dendritic spines. Neuroscience 13:1189-1215.

Furuta T, Tomioka R, Taki K, Nakamura K, Tamamaki N, Kaneko T (2001) In vivo transduction of central neurons using recombinant Sindbis virus: Golgi-like labeling of dendrites and axons with membrane-targeted fluorescent proteins. J Histochem Cytochem 49:1497-1508.

Gauthier J, Parent M, Lévesque M, Parent A (1999) The axonal arborization of single nigrostriatal neurons in rats. Brain Res 834:228-232.

Gauthier-Campbell C, Bredt DS, Murphy TH, El-Husseini Ael-D (2004) Regulation of dendritic branching and filopodia formation in hippocampal neurons by specific acylated protein motifs. Mol Biol Cell 15:2205-2217.

Gerfen CR (1984) The neostriatal mosaic: compartmentalization of corticostriatal input and striatonigral output systems. Nature 311:461-464.

Gerfen CR (1985) The neostriatal mosaic. I. Compartmental organization of projections from the striatum to the substantia nigra in the rat. J Comp Neurol 236:454-476.

Gerfen CR (1989) The neostriatal mosaic: striatal patch-matrix organization is related to cortical lamination. Science 246:385-388.

Gerfen CR, Baimbridge KG, Miller JJ (1985) The neostriatal mosaic: compartmental distribution of calcium-binding protein and parvalbumin in the basal ganglia of the rat and monkey. Proc Natl Acad Sci U S A $82: 8780-8784$.

Gerfen CR, Herkenham M, Thibault J (1987a) The neostriatal mosaic: II. Patch- and matrix-directed mesostriatal dopaminergic and nondopaminergic systems. J Neurosci 7:3915-3934.

Gerfen CR, Baimbridge KG, Thibault J (1987b) The neostriatal mosaic: III. Biochemical and developmental dissociation of patch-matrix mesostriatal systems. J Neurosci 7:3935-3944.

Grace AA, Bunney BS (1983) Intracellular and extracellular electrophysiology of nigral dopaminergic neurons-3. Evidence for electrotonic coupling. Neuroscience 10:333-348.

Graybiel AM, Ragsdale CW Jr (1978) Histochemically distinct compartments in the striatum of human, monkeys, and cat demonstrated by acetylthiocholinesterase staining. Proc Natl Acad Sci U S A 75:5723-5726.

Halliday GM, Törk I (1986) Comparative anatomy of the ventromedial mesencephalic tegmentum in the rat, cat, monkey and human. J Comp Neurol 252:423-445.

Herkenham M, Pert CB (1981) Mosaic distribution of opiate receptors, parafascicular projections and acetylcholinesterase in rat striatum. Nature 291:415-418.

Hirsch E, Graybiel AM, Agid YA (1988) Melanized dopaminergic neurons are differentially susceptible to degeneration in Parkinson's disease. Nature 334:345-348.

Ito T, Hioki H, Nakamura K, Tanaka Y, Nakade H, Kaneko T, Iino S, Nojyo Y (2007) Gamma-aminobutyric acid-containing sympathetic preganglionic neurons in rat thoracic spinal cord send their axons to the superior cervical ganglion. J Comp Neurol 502:113-125.

Jimenez-Castellanos J, Graybiel AM (1987) Subdivisions of the dopaminecontaining A8-A9-A10 complex identified by their differential mesostriatal innervation of striosomes and extrastriosomal matrix. Neuroscience 23:223-242.

Joel D, Weiner I (2000) The connections of the dopaminergic system with the striatum in rats and primates: an analysis with respect to the functional and compartmental organization of the striatum. Neuroscience 96:451-474.

Johnston JG, Gerfen CR, Haber SN, van der Kooy D (1990) Mechanisms of striatal pattern formation: conservation of mammalian compartmentalization. Brain Res Dev Brain Res 57:93-102.

Juraska JM, Wilson CJ, Groves PM (1977) The substantia nigra of the rat: a Golgi study. J Comp Neurol 172:585-600. 
Kaneko T, Caria MA, Asanuma H (1994) Information processing within the motor cortex. II. Intracortical connections between neurons receiving somatosensory cortical input and motor output neurons of the cortex. J Comp Neurol 345:172-184.

Kaneko T, Minami M, Satoh M, Mizuno N (1995) Immunocytochemical localization of mu-opioid receptor in the rat caudate-putamen. Neurosci Lett 184:149-152.

Langer LF, Graybiel AM (1989) Distinct nigrostriatal projection systems innervate striosomes and matrix in the primate striatum. Brain Res 498:344-350.

Lee T, Kaneko T, Taki K, Mizuno N (1997) Preprodynorphin-, preproenkephalin-, and preprotachykinin-expressing neurons in the rat neostriatum: an analysis by immunocytochemistry and retrograde tracing. J Comp Neurol 386:229-244.

Lévesque M, Parent A (2005) The striatofugal fiber system in primates: a reevaluation of its organization based on single-axon tracing studies. Proc Natl Acad Sci U S A 102:11888-11893.

Lundstrom K (1999) Alphaviruses as tools in neurobiology and gene therapy. J Recept Signal Transduct Res 19:673-686.

Nakamura K, Matsumura K, Kaneko T, Kobayashi S, Katoh H, Negishi M (2002) The rostral raphe pallidus nucleus mediates pyrogenic transmission from the preoptic area. J Neurosci 22:4600-4610.

Nakamura K, Matsumura K, Hübschle T, Nakamura Y, Hioki H, Fujiyama F, Boldogköi Z, König M, Thiel HJ, Gerstberger R, Kobayashi S, Kaneko T (2004) Identification of sympathetic premotor neurons in medullary raphe regions mediating fever and other thermoregulatory functions. J Neurosci 24:5370-5380.

Nakamura Y, Nakamura K, Matsumura K, Kobayashi S, Kaneko T, Morrison SF (2005) Direct pyrogenic input from prostaglandin EP3 receptorexpressing preoptic neurons to the dorsomedial hypothalamus. Eur J Neurosci 22:3137-3146.

Oorschot DE (1996) Total number of neurons in the neostriatal, pallidal, subthalamic, and substantia nigral nuclei of the rat basal ganglia: a stereological study using the cavalieri and optical disector methods. J Comp Neurol 366:580-599.
Pickel VM, Beckley SC, Joh TH, Reis DJ (1981) Ultrastructural immunocytochemical localization of tyrosine hydroxylase in the neostriatum. Brain Res 225:373-385.

Piper RC, Slot JW, Li G, Stahl PD, James DE (1994) Recombinant Sindbis virus as an expression system for cell biology. Methods Cell Biol 43:55-78

Prensa L, Parent A (2001) The nigrostriatal pathway in the rat: a single-axon study of the relationship between dorsal and ventral tier nigral neurons and the striosome/matrix striatal compartments. J Neurosci 21:7247-7260.

Preston RJ, McCrea RA, Chang HT, Kitai ST (1981) Anatomy and physiology of substantia nigra and retrorubral neurons studied by extra- and intracellular recording and by horseradish peroxidase labeling. Neuroscience 6:331-344.

Schlesinger S (1993) Alphaviruses-vectors for the expression of heterologous genes. Trends Biotechnol 11:18-22.

Schultz W (1998) Predictive reward signal of dopamine neurons. J Neurophysiol 80:1-27.

Strauss JH, Strauss EG (1994) The alphaviruses: gene expression, replication, and evolution. Microbiol Rev 58:491-562.

Tamamaki N, Nakamura K, Furuta T, Asamoto K, Kaneko T (2000) Neurons in Golgi-stain-like images revealed by GFP-adenovirus infection in vivo. Neurosci Res 38:231-236.

Tepper JM, Sawyer SF, Groves PM (1987) Electrophysiologically identified nigral dopaminergic neurons intracellularly labeled with HRP: lightmicroscopic analysis. J Neurosci 7:2794-2806.

Vandecasteele M, Glowinski J, Venance L (2005) Electrical synapses between dopaminergic neurons of the substantia nigra pars compacta. J Neurosci 25:291-298.

Xiong C, Levis R, Shen P, Schlesinger S, Rice CM, Huang HV (1989) Sindbis virus: an efficient, broad host range vector for gene expression in animal cells. Science 243:1188-1191.

Zahm DS (1992) An electron microscopic morphometric comparison of tyrosine hydroxylase immunoreactive innervation in the neostriatum and the nucleus accumbens core and shell. Brain Res 575:341-346. 